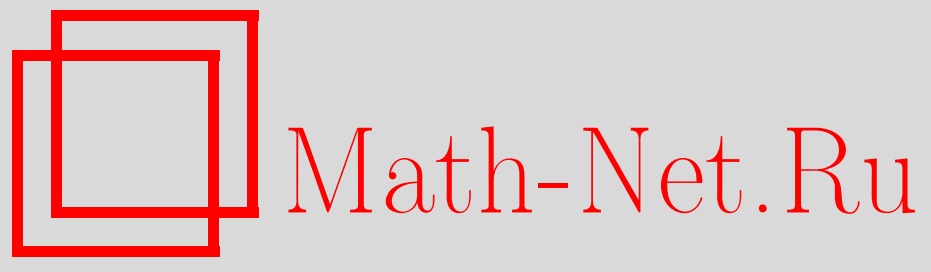

Е. В. Никитин, Классы Бесова на бесконечномерных пространствах, Матем. заметки, 2013, том 93, выпуск 6, 951-953

DOI: https://doi.org/10.4213/mzm10248

Использование Общероссийского математического портала Math-Net.Ru подразумевает, что вы прочитали и согласны с пользовательским соглашением http://www . mathnet.ru/rus/agreement

Параметры загрузки:

IP : 34.229 .108 .108

26 апреля 2023 г., 09:52:56

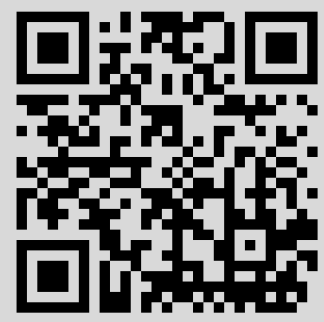




\section{Классы Бесова на бесконечномерных пространствах}

\section{Е. В. Никитин}

В этой работе вводятся пространства Бесова на бесконечномерных пространствах с гауссовской мерой аналогично прямому определению через интегральное условие, известному в конечномерном случае (см. [1], [2]). Основной результат - теорема вложения для введенных пространств.

Для всякой функции $u$ на $\mathbb{R}^{n}$ и всякого вектора $h \in \mathbb{R}^{n}$ положим $\Delta_{h} u(x)=u(x)$ $u(x-h)$. При $s>0, p>1$ и $q \geqslant 1$ класс Бесова функций на пространстве $\mathbb{R}^{n}$ определяется как множество всех функций $u \in L^{p}\left(\mathbb{R}^{n}\right)$, для которых

$$
\int_{\mathbb{R}^{n}}|h|^{-s-n}\left\|\left(\Delta_{h}\right)^{m} u\right\|_{p}^{q}(d h)<\infty .
$$

Пусть $X$ - локально выпуклое пространство с топологическим сопряженным $X^{*}$. Вероятностная мера $\gamma$, определенная на $\sigma$-алгебре $\mathcal{E}(X)$, порожденной $X^{*}$, называется гауссовской (см. [3]), если для всякого функционала $f \in X^{*}$ мера $\gamma \circ f^{-1}$ является гауссовской на прямой. Основной пример - стандартная гауссовская мера на $X=\mathbb{R}^{\infty}$ (счетная степень прямой), равная счетной степени стандартной гауссовской меры на прямой.

Класс гладких цилиндрических функций $\mathcal{F} \mathcal{C}^{\infty}$ состоит из функций вида

$$
f(x)=g\left(l_{1}(x), \ldots, l_{n}(x)\right), \quad \text { где } \quad g \in C_{\mathrm{b}}^{\infty}\left(\mathbb{R}^{n}\right), \quad l_{i} \in X^{*} .
$$

Если $X=\mathbb{R}^{\infty}$, то это функции от конечного числа переменных с ограниченными производными всех порядков.

Для $p \geqslant 1$ и $r \in \mathbb{N}$ классы Соболева $W^{p, r}(\gamma)$ определяются как пополнение пространства гладких цилиндрических функций $\mathcal{F} \mathcal{C}^{\infty}$ по соболевской норме $\|\cdot\|_{W^{p, r}}$, определяемой по формуле

$$
\|f\|_{W^{p, r}}=\sum_{k=0}^{r}\left\|D_{H}^{k} f\right\|_{L^{p}\left(\gamma, \mathcal{H}_{k}\right)} .
$$

Здесь $D_{H}^{k} f$ является отображением из $X$ в пространство $k$-линейных отображений Гильберта-Шмидта из $H(\gamma)$ в $\mathbb{R}^{1} ; D_{H} f$ для функций из $\mathcal{F C} \mathcal{C}^{\infty}$ определяется для $h \in H(\gamma)$ по формуле

$$
D_{H} f(x) h=\lim _{t \rightarrow 0} t^{-1}(f(x+t h)-f(x)) .
$$

Производные более высокого порядка $D_{H}^{k} f$ определяются индуктивно как $D_{H}\left(D_{H}^{k-1} f\right)$. Подробнее о классах Соболева в бесконечномерных пространствах см. [3]-[7].

Пусть теперь на локально выпуклом пространстве $X$ задана центрированная радоновская гауссовская мерой $\gamma$ (см. [3]). Пространство Камерона-Мартина $H=H(\gamma)$ состоит из всех векторов $h \in X$, имеющих конечную норму

$$
|h|_{H}:=\sup \left\{l(h): l \in X^{*},\|l\|_{L^{2}(\gamma)} \leqslant 1\right\} .
$$

Эта норма порождается скалярным произведением $\langle k, h\rangle$, которое можно задать так: для всякого $h \in H$ найдется элемент $l_{h}$ из замыкания $X^{*}$ в $L^{2}(\gamma)$, удовлетворяющий тождеству

$$
l(h)=\int_{X} l_{h} l d \gamma, \quad l \in X^{*} .
$$

Работа выполнена при поддержке Российского фонда фундаментальных исследований (гранты №№ 10-01-00518, 11-01-90421-У кр-ф-а).

DOI: $10.4213 / \mathrm{mzm} 10248$ 
Тогда $\langle k, h\rangle=\left(l_{h}, l_{k}\right)_{L^{2}(\gamma)}$. Для стандартной гауссовской меры на $\mathbb{R}^{\infty}$ получаем $H=\ell^{2}$ с обычным скалярным произведением.

Положим $\Delta_{h} u(x)=u(x)-u(x-h)$, где $h \in H(\gamma)$. Норму в $L^{p}(\gamma)$ будем обозначать через $\|\cdot\|_{p}$.

ОПРеДЕЛЕниЕ 1 . Пусть $0<s<\infty, 1<p<\infty, 1 \leqslant q<\infty, \nu$ - борелевская вероятностная мера на $H(\gamma)$ и $m$ - наименьшее целое, большее $s$. Kласс Бесова $B^{s ; p, q, \nu}(\gamma)$ состоит из всех таких функций $u \in L^{p}(\gamma)$, что

$$
\int_{H}|h|_{H}^{-s}\left\|\left(\Delta_{h}\right)^{m} u\right\|_{p}^{q} \nu(d h)<\infty .
$$

Норма в $B^{s ; p, q, \nu}(\gamma)$ задается формулой

$$
\|u\|_{s ; p, q, \nu}:=\|u\|_{p}+\left(\int_{H}|h|_{H}^{-s}\left\|\left(\Delta_{h}\right)^{m} u\right\|_{p}^{q} \nu(d h)\right)^{1 / q} .
$$

Отметим, что определяемые так пространства существенно зависят не только от трех числовых параметров, как обычные классы Бесова, но и от дополнительного параметра, которым является мера на пространстве Камерона-Мартина.

Лемма 1. Пространства $B^{s ; p, q, \nu}(\gamma)$ являются банаховыми.

ДоказАтельство. Пусть последовательность функций $\left\{\varphi_{j}\right\}_{j=0}^{\infty}$ из класса Бесова $B^{s ; p, q, \nu}(\gamma)$ фундаментальна по норме $\|\cdot\|_{s ; p, q, \nu}$. Тогда она фундаментальна и по норме в $L^{p}(\gamma)$ и в силу полноты $L^{p}(\gamma)$ сходится к некоторой функции $\varphi$ из $L^{p}(\gamma)$. Перейдя к подпоследовательности, можно считать, что $\left\{\varphi_{j}\right\}$ сходится к $\varphi$ почти всюду. Из теоремы Фату ясно, что $\varphi \in B^{s ; p, q, \nu}(\gamma)$. Теперь достаточно доказать, что какая-нибудь подпоследовательность в $\left\{\varphi_{j}\right\}$ сходится к $\varphi$ по норме $B^{s ; p, q, \nu}(\gamma)$. Для этого выберем еще одну подпоследовательность, обозначая ее снова через $\left\{\varphi_{j}\right\}$, для которой

$$
\left\|\varphi_{k}-\varphi_{j}\right\|_{s ; p, q, \nu} \leqslant 2^{-j} \quad \text { при } \quad k>j .
$$

Тогда $\lim _{k \rightarrow \infty}\left(\varphi_{k}-\varphi_{j}\right)=\varphi-\varphi_{j}$ почти всюду. Положив

$$
Q_{j}:=\left|\left(\Delta_{h}\right)^{m}\left(\varphi-\varphi_{j}\right)\right|^{p}, \quad Q_{j, k}=\left|\left(\Delta_{h}\right)^{m}\left(\varphi_{k}-\varphi_{j}\right)\right|^{p},
$$

получим $Q_{j}=\lim _{k \rightarrow \infty} Q_{j, k}$ почти всюду. Теперь оценим второе слагаемое в выражении для нормы $\left\|\varphi-\varphi_{j}\right\|_{s ; p, q, \nu}$, дважды применяя лемму Фату:

$$
\begin{aligned}
\int_{H} \mid & \left.h\right|_{H} ^{-s}\left(\int_{X} Q_{j} d \gamma\right)^{q / p} d \nu \leqslant \int_{H}|h|_{H}^{-s} \liminf _{k \rightarrow \infty}\left(\int_{X} Q_{j, k} d \gamma\right)^{q / p} d \nu \\
& \leqslant \liminf _{k \rightarrow \infty} \int_{H}|h|_{H}^{-s}\left(\int_{X} Q_{j, k} d \gamma\right)^{q / p} d \nu \leqslant \liminf _{k \rightarrow \infty}\left\|\varphi_{k}-\varphi_{j}\right\|_{s ; p, q, \nu}^{q} \leqslant 2^{-q j} .
\end{aligned}
$$

Таким образом, $\left\|\varphi-\varphi_{j}\right\|_{s ; p, q, \nu} \rightarrow 0$ при $j \rightarrow \infty$. Лемма доказана.

Теорема 1. Пусть вероятностная мера $\nu$ на $H(\gamma)$ такова, что функиия $\exp \left(M|h|_{H}^{2}\right)$ интегрируема по у при некотором $M>q /\left(2\left(p_{1}-p\right)\right)$. Тогда при $p_{1}>p u 0<s \leqslant 1$ имеем

$$
W^{p_{1}, 1}(\gamma) \subset B^{s ; p, q, \nu}(\gamma)
$$

ДокАзАтеЛЬСтво. Для всякой функции $f \in W^{p, 1}$ при каждом $h \in H$ почти всюду верно равенство

$$
f(x+h)-f(x)=\int_{0}^{1}\left\langle D_{H} f(x+t h), h\right\rangle d t .
$$


Оценим $L^{p}$-норму $\Delta_{h} f$ (возведенную в степень $p$ ). Чтобы занести степень $p$ внутрь интеграла, применим неравенство Йенсена:

$$
\begin{aligned}
\int_{X}|f(x+h)-f(x)|^{p} \gamma(d x) & \leqslant|h|^{p} \int_{X}\left(\int_{0}^{1}\left|D_{H} f(x+t h)\right|_{H} d t\right)^{p} \gamma(d x) \\
& \leqslant|h|^{p} \int_{0}^{1} \int_{X}\left|D_{H} f(x+t h)\right|_{H}^{p} \gamma(d x) d t .
\end{aligned}
$$

В последнем интеграле сделаем замену переменных, взяв $x+t h$ за новую переменную и применив формулу Камерона-Мартина, согласно которой при сдвиге гауссовской меры $\gamma$ на вектор $h$ из пространства Камерона-Мартина получается эквивалентная исходной мера с плотностью $\exp \left(l_{h}-|h|_{H}^{2} / 2\right)$. Получаем соотношения

$$
\begin{aligned}
|h|^{p} & \int_{0}^{1} \int_{X}\left|D_{H} f(x+t h)\right|_{H}^{p} \gamma(d x) d t=|h|^{p} \int_{0}^{1} \int_{X}\left|D_{H} f(x)\right|_{H}^{p} e^{t l_{h}-\left(t^{2} / 2\right)|h|^{2}} \gamma(d x) d t \\
& \leqslant|h|^{p} \int_{0}^{1}\left(\int_{X}\left|D_{H} f(x)\right|_{H}^{p r} \gamma(d x)\right)^{1 / r}\left(\int_{X} e^{m\left(t l_{h}-\left(t^{2} / 2\right)|h|^{2}\right)} \gamma(d x)\right)^{1 / m} d t \\
& =|h|^{p}\left(\int_{X}\left|D_{H} f(x)\right|_{H}^{p r} \gamma(d x)\right)^{1 / r} \int_{0}^{1} e^{((m-1) / 2)|h|^{2} t^{2}} d t .
\end{aligned}
$$

Чтобы отделить плотность, возникающую из формулы Камерона-Мартина, мы воспользовались неравенством Гёльдера для некоторых чисел $r>1, m>1$ таких, что $r^{-1}+m^{-1}=1$, выбираемых далее. Эта часть явно интегрируется по $X$. Подставив полученную оценку в выражение для нормы Бесова, получаем

$$
\begin{aligned}
\int_{H}|h|^{-s}\left\|\Delta_{h} f\right\|_{L^{p}}^{q} \nu(d h) & \\
& \leqslant\left(\int_{X}\left|D_{H} f(x)\right|_{H}^{p r} \gamma(d x)\right)^{q /(p r)} \int_{H}|h|^{1-s}\left(\int_{0}^{1} e^{((m-1) / 2)|h|^{2} t^{2}} d t\right)^{q / p} \nu(d h) \\
& \leqslant\left(\int_{X}\left|D_{H} f(x)\right|_{H}^{p_{1}} \gamma(d x)\right)^{q / p_{1}} \int_{H}|h|^{1-s} e^{\left(q /\left(2\left(p_{1}-p\right)\right)\right)|h|^{2}} \nu(d h) \\
& \leqslant C(s, M) \int_{H} e^{M|h|^{2}} \nu(d h)\left(\int_{X}\left|D_{H} f(x)\right|_{H}^{p_{1}} \gamma(d x)\right)^{q / p_{1}} .
\end{aligned}
$$

Положив $r=p_{1} / p$, получаем, что для любой функции $f \in W^{p_{1}, 1}$ конечна норма $\|f\|_{B^{p, q, 1, \nu}}$, а это дает нужное вложение с оценкой для норм. Теорема доказана.

\section{СПИСОК ЦИТИРОВАННОЙ ЛИТЕРАТУРЫ}

[1] О. В. Бесов, В. П. Ильин, С. М. Никольский, Интегральные представления функиий и теоремы вложсения, Наука, M., 1996. [2] R. A. Adams, J. J. F. Fournier, Sobolev Spaces, Pure Appl. Math. (Amst.), 140, Academic Press, New York, 2003. [3] В. И. Богачев, Гауссовские меры, Наука, M., 1997. [4] V. I. Bogachev, J. Math. Sci. (New York), 87:4 (1997), 3577-3731. [5] V. I. Bogachev, Differentiable Measures and the Malliavin Calculus, Math. Surveys Monogr., 164, Amer. Math. Soc., Providence, RI, 2010. [6] V. Bogachev, E. Mayer-Wolf, 167:1 (1999), 1-68. [7] I. Shigekawa, Stochastic Analysis, Transl. Math. Monogr., 224, Amer. Math. Soc., Providence, RI, 2004.

\section{Е. В. Никитин}

